\title{
PD-L1 on host cells is essential for PD-L1 blockade- mediated tumor regression
}

\author{
Haidong Tang, ${ }^{1}$ Yong Liang, ${ }^{2}$ Robert A. Anders, ${ }^{3}$ Janis M. Taube, ${ }^{3,4}$ Xiangyan Qiu, ${ }^{1}$ Aditi Mulgaonkar, ${ }^{5}$ Xin Liu, ${ }^{6}$ \\ Susan M. Harrington, ${ }^{6}$ Jingya Guo, ${ }^{2}$ Yangchun Xin, ${ }^{5}$ Yahong Xiong, ${ }^{7}$ Kien Nham, ${ }^{5}$ William Silvers, ${ }^{5}$ Guiyang Hao, ${ }^{5}$ \\ Xiankai Sun, ${ }^{8}$ Mingyi Chen, ${ }^{1}$ Raquibul Hannan, ${ }^{9}$ Jian Qiao, ${ }^{1}$ Haidong Dong, ${ }^{6}$ Hua Peng, ${ }^{2}$ and Yang-Xin Fu ${ }^{1,2}$

\begin{abstract}
'Department of Pathology, University of Texas (UT) Southwestern Medical Center, Dallas, Texas, USA. ${ }^{2}$ Chinese Academy of Sciences Key Laboratory for Infection and Immunity, Institute of Biophysics, Chinese Academy of Sciences, Beijing, China. ${ }^{3}$ Department of Pathology, Johns Hopkins University School of Medicine, Baltimore, Maryland, USA. ${ }^{4}$ Department of Dermatopathology and the Bloomberg-Kimmel Institute of Cancer Immunotherapy and ${ }^{5}$ Department of Radiology, UT Southwestern Medical Center, Dallas, Texas, USA. ${ }^{6}$ Departments of Urology and Immunology, College of Medicine, Mayo Clinic, Rochester, Minnesota, USA. `Department of Applied Chemistry, College of Materials and Energy, South China Agricultural University, Guangzhou, Guangdong, China. ${ }^{8}$ Department of Radiology and Advanced Imaging Research Center and ${ }^{9}$ Department of Radiation Oncology, UT Southwestern Medical Center, Dallas, Texas, USA.
\end{abstract}

\begin{abstract}
Programmed death-ligand 1 (PD-L1) expression on tumor cells is essential for T cell impairment, and PD-L1 blockade therapy has shown unprecedented durable responses in several clinical studies. Although higher expression of PD-L1 on tumor cells is associated with a better immune response after Ab blockade, some PD-L1-negative patients also respond to this therapy. In the current study, we explored whether PD-L1 on tumor or host cells was essential for anti-PD-L1-mediated therapy in 2 different murine tumor models. Using real-time imaging in whole tumor tissues, we found that anti-PD-L1 Ab accumulates in tumor tissues, regardless of the status of PD-L1 expression on tumor cells. We further observed that, while PD-L1 on tumor cells was largely dispensable for the response to checkpoint blockade, PD-L1 in host myeloid cells was essential for this response. Additionally, PD-L1 signaling in defined antigen-presenting cells (APCs) negatively regulated and inhibited T cell activation. PD-L1 blockade inside tumors was not sufficient to mediate regression, as limiting $\mathrm{T}$ cell trafficking reduced the efficacy of the blockade. Together, these findings demonstrate that PD-L1 expressed in APCs, rather than on tumor cells, plays an essential role in checkpoint blockade therapy, providing an insight into the mechanisms of this therapy.
\end{abstract}

\section{Introduction}

The programmed death-1 receptor/programmed death-ligand 1 (PD-1/PD-L1) signaling pathway plays an important role in tumor evasion of host immune responses (1). Recent clinical trials with PD-1/PD-L1 blockade therapy have shown unprecedented durable response in patients with a variety of cancers $(2,3)$. However, only a minority of patients benefits from such therapy. Thus, it has become a top priority to identify biomarkers that can predict patient responses (4). It has been suggested that PD-L1 on tumor cells plays an important role in preventing $\mathrm{T}$ cell-mediated killing. Higher expression of PD-L1 on tumor cells has been shown to be associated with a better immune response after checkpoint blockade (5-8). However, clinical responses were also observed in some patients that were negative for PD-L1 in tumors $(9,10)$. The contributions of PD-L1 expression from relevant cells to inhibition of $\mathrm{T}$ cell responses remains unknown (11-13).

Expression of PD-L1 is limited in normal tissues (14). In contrast, many tumor cells overexpress PD-L1 as a strategy to evade

\section{Related Commentary: p. 570}

Authorship note: $\mathrm{H}$. Tang and Y. Liang contributed equally to this work. Conflict of interest: The authors have declared that no conflict of interest exists. Submitted: July 5, 2017; Accepted: November 16, 2017. Reference information: J Clin Invest. 2018;128(2):580-588. https://doi.org/10.1172/JCI96061. immune responses. Interestingly, PD-L1 can be upregulated on many cells when stimulated by inflammatory cytokines, especially IFNs $(4,14-16)$. In the context of tumor microenvironment, cells including macrophages, DCs, myeloid-derived suppressor cells (MDSCs), Tregs, and endothelial cells, can upregulate PD-L1 due to inflammation responses (15). However, the relative contributions of PD-L1 in these cells are unclear. Our previous study compared a series of well-established mouse tumor lines for their PD-L1 expression and responsiveness to anti-PD-L1 treatment (17). Some tumor cells express similar high levels of PD-L1, but respond differently to PD-L1 blockade. This raises the possibility that PD-L1 expressed in tumor cells is not sufficient for the response to checkpoint blockade therapy. In the current study, we sought to define the roles of PD-L1 from tumor versus host cells during checkpoint blockade therapy.

\section{Results}

PD-L1 on tumor cells is dispensable for checkpoint blockade therapy. To test this hypothesis, tissues were collected from MC38 tumor-bearing mice and the PD-L1 expression profiles were evaluated by flow cytometry (Figure 1A). Surprisingly, myeloid cells, including macrophages $\left(\mathrm{CD} 11 \mathrm{~b}^{+} \mathrm{F} 4 / 80^{+}\right)$, MDSCs $\left(\mathrm{CD} 11 \mathrm{~b}^{+} \mathrm{Gr}-1^{+}\right)$, and DCs $\left(C D 11 c^{+}\right)$, expressed much higher levels of PD-L1 than tumor and stromal (CD45-) cells (Figure 1, B-D). Furthermore, myeloid cells in tumor-draining lymph nodes (dLNs) expressed even higher PD-L1 than these cells in tumor tissues (Figure 1, C 
A $\quad M \phi$
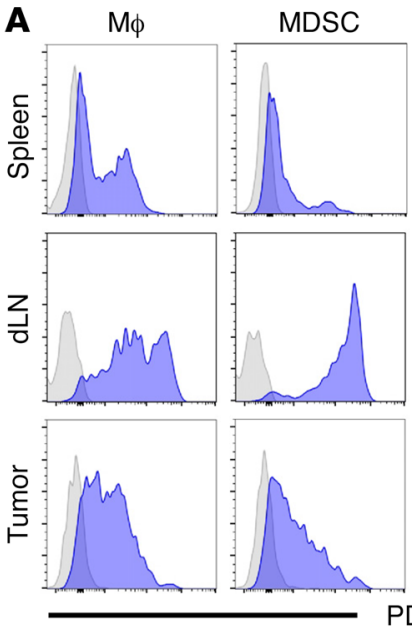

E

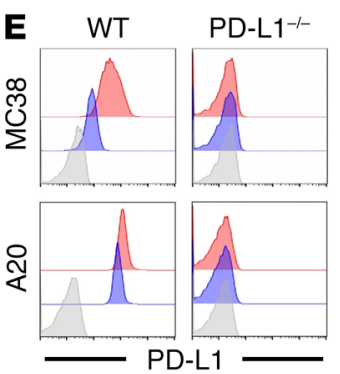

- Control

$-\alpha-P D-L 1$

$-\alpha-P D-L 1+$ IFN- $\gamma$
CD45-

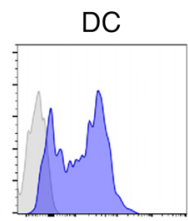

- FMO

- $\alpha$-PD-L
B

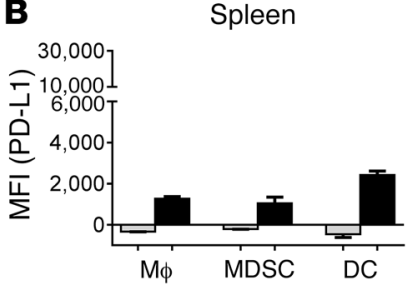

C

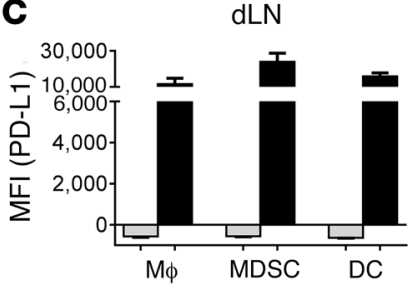

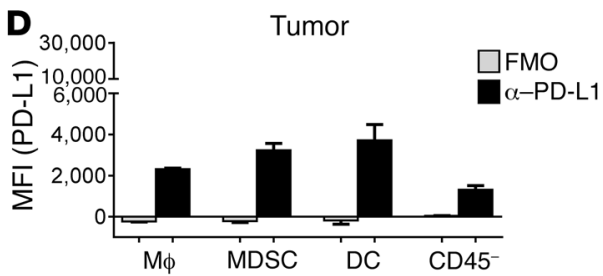

PD-L1
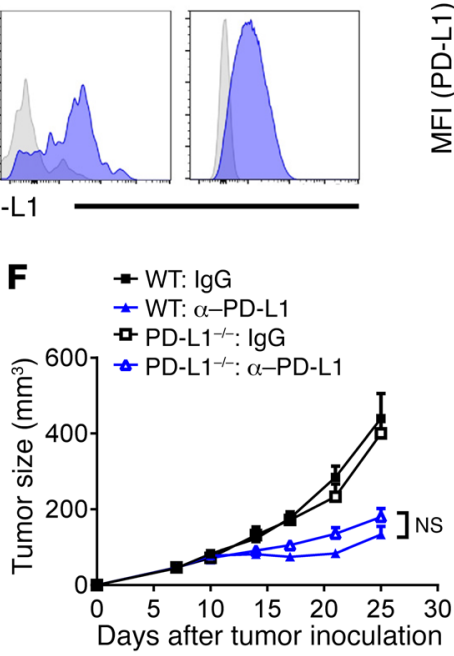

\section{G}
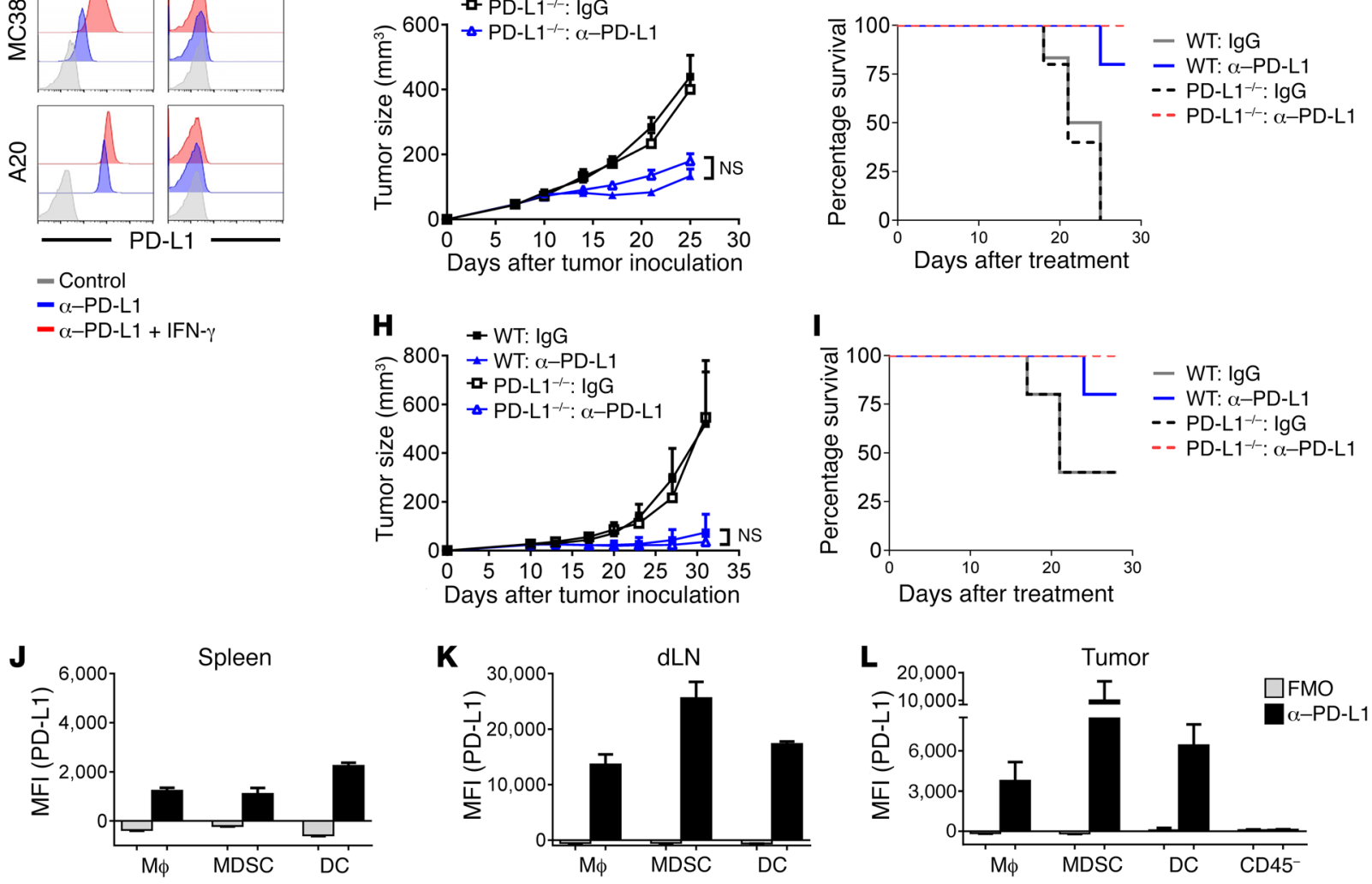

Figure 1. Tumor-expressed PD-L1 is dispensable for responses to checkpoint blockade therapy. (A) C57BL/6 mice were inoculated with $1 \times 10^{6} \mathrm{MC} 38$ cells. Spleen, dLN, and tumor tissues were collected on day 22. PD-L1 expression was measured by flow cytometry. FMO, fluorescence minus one. (B-D) Mean fluorescent intensities (MFIs) of PD-L1 staining in spleen (B), dLN (C), and tumor (D) are shown ( $n=3$ per group). (E) PD-L1 expression in MC38.WT, MC38. PD-L1/-1- A20.WT, and A20.PD-L1/-/- cells was measured by flow cytometry. To induce PD-L1 expression, cells were treated with $500 \mathrm{U} / \mathrm{ml}$ IFN- $\gamma$ for 24 hours. (F and G) C57BL/ 6 mice ( $n=5$ or 6 ) were inoculated with $1 \times 10^{6}$ MC38.WT or MC38.PD-L1 ${ }^{-1-}$ cells. After tumors were established, mice were treated with $200 \mu$ g anti-PD-L1 on days 7, 10, and 13. Tumor growth (F) and survival curve (G) are shown. (H and I) BALB/c mice ( $n=5)$ were inoculated with $3 \times 10^{6}$ A20.WT or A20. PD-L1 ${ }^{-/-}$cells. Mice were treated with $200 \mu \mathrm{g}$ anti-PD-L1 on days 10 and 13 . Tumor growth (H) and survival curve (I) are shown. (J-L) Tissues were collected from MC38.PD-L1 ${ }^{-/-}$tumor-bearing mice. Mean fluorescent intensities of PD-L1 staining in spleen (J), dLN $(\mathbf{K})$, and tumor (L) are shown ( $\left.n=3\right)$. Data indicate mean \pm SEM and are representative of at least 2 independent experiments. Statistical analysis was performed using an unpaired Student's 2 -tailed $t$ test.

and D). In contrast, PD-L1 expression in lymphocytes, including $\mathrm{CD}^{+} \mathrm{T}$ cells, $\mathrm{CD} 4^{+} \mathrm{T}$ cells, and B cells, was much lower (Supplemental Figure 1, A-D; supplemental material available online with this article; https://doi.org/10.1172/JCI96061DS1). Consistently, some recent studies showed that most tumor cells do not express high levels of PD-L1, while adjacent cells around tumor cells and inflammation cells express higher levels of PD-L1 $(7,8,18)$. Taken together, these data raise the possibility that higher levels of 
A
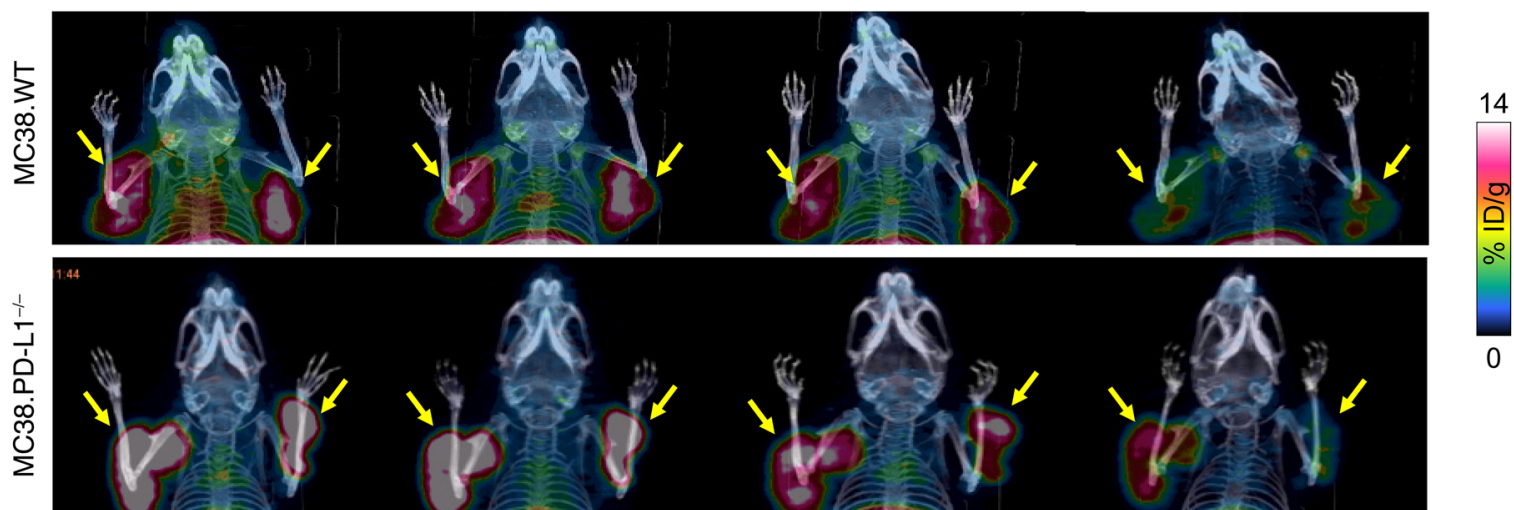

B

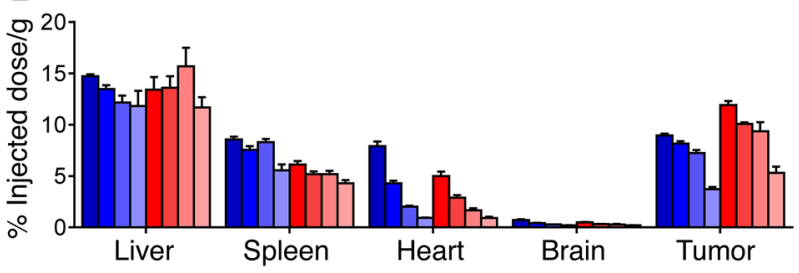

D

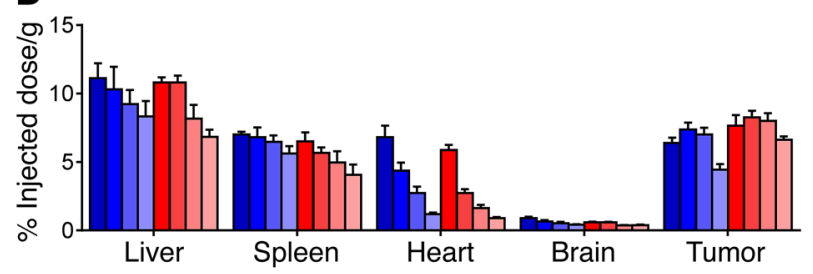

C

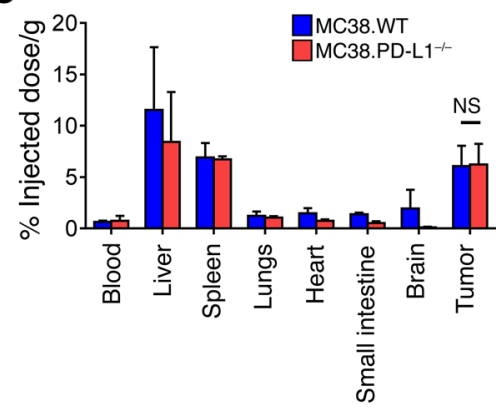

Figure 2. Anti-PD-L1 Ab targeting to tumor tissue is independent of tumor PD-L1. (A) MC38.WT or MC38.PD-L1/- tumor-bearing mice were injected with $50 \mu \mathrm{Ci}$ of ${ }^{89} \mathrm{Zr}$-radiolabeled deferoxamine-conjugated anti-PD-L1 ( ${ }^{89} \mathrm{Zr}$-anti-PD-L1) Abs ( $n=3$ per group). Ab distribution was imaged by PET/CT on 1, 2, 3 , and 6 d.p.i. One representative mouse from each group is shown. Yellow arrows indicate tumors. (B) The uptake of ${ }^{89} \mathrm{Zr}$-anti-PD-L1 Abs in MC38 tumors was measured by PET/CT and quantitated in various organs on 1, 2, 3, and 6 d.p.i. (C) Ex vivo biodistribution of ${ }^{89} \mathrm{Zr}$-anti-PD-L1 Ab uptake on 6 d.p.i. is shown. (D) The uptake of ${ }^{89} \mathrm{Zr}$-anti-PD-L1 Abs was measured and quantitated by PET/CT in mice bearing A20.WT or A20.PD-L1 ${ }^{-/-}$tumors ( $n=3$ per group). Statistical analysis was performed using an unpaired Student's 2-tailed $t$ test.

PD-L1 on myeloid cells might play more important roles in controlling T cell responses.

Even though PD-L1 in tumor cells could positively correlate with overall patient response to PD-1/PD-L1 blockade, it is difficult to determine essential or dominant roles of PD-L1 on tumor versus host cells through current preclinical and clinical studies. To investigate the role of tumor-expressed PD-L1, we knocked out PD-L1 in tumor cells by clustered, regularly interspaced, short palindromic repeats-associated nuclease Cas9 (CRISPR/Cas9) technology. Knockout tumor cells lacked PD-L1 expression, as measured by flow cytometry (Figure 1E). IFNs are strong inducers of PD-L1 (19). When stimulated by IFN- $\gamma$, WT MC38 (MC38.WT) cells upregulated PD-L1 expression while PD-L1-knockout MC38 (MC38.PD-L1 ${ }^{-/}$) cells remained negative, indicating a complete ablation of gene expression (Figure 1E). When inoculated into the WT host, MC38.PD-L1 ${ }^{-/-}$tumors grew similarly to WT tumor (Figure $1 \mathrm{~F}$ ). Surprisingly, response of $\mathrm{MC} 38 . \mathrm{PD}-\mathrm{L}^{-/-}$tumor to PD-L1 blockade therapy was as good as that of WT tumor (Figure $1, F$ and $G$ ). Similar results were observed using PD-L1-deficient A20 tumor (Figure 1, E, H, and I). Both PD-L1-deficient MC38 and
A20 tumors also responded to PD-1 blockade therapy well (Supplemental Figure 2, A and B). To find out whether there are differences in host PD-L1 expression between MC38.WT and MC38. $\mathrm{PD}-\mathrm{L1}^{-/}$tumors, tissues were collected and PD-L1 expression was evaluated by flow cytometry. Interestingly, while tumor cells completely lost PD-L1 expression, the levels of PD-L1 in myeloid cells from MC38.PD-L1 ${ }^{-/}$tumor-bearing mice were similar to their counterparts in WT tumor-bearing mice (Supplemental Figure 1E and Figure 1, J-L). Collectively, these data suggest that PD-L1 on tumor cells is not essential for the response to PD-L1 blockade in these models. It is possible that myeloid cell-expressed PD-L1 is sufficient to limit immune responses, and thus myeloid cells may mediate the response to checkpoint blockade therapy.

Anti-PD-L1 Ab targets to tumor tissue regardless of the status of tumor-expressed PD-L1. Lack of PD-L1 expression on a biopsy specimen cannot exclude PD-L1 expression in different areas of tumor tissues or subsequent expression after sampling. Additionally, the lack of approaches that can detect PD-L1 in real time within in vivo, whole tumor tissues during PD-L1 therapy might complicate clinical interpretations of PD-L1 as biomark- 
A

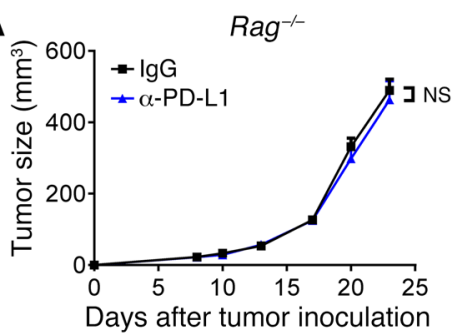

C

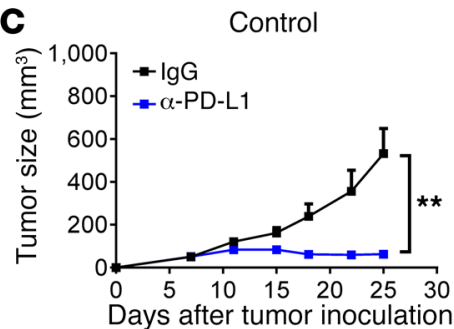

$\mathbf{F}$

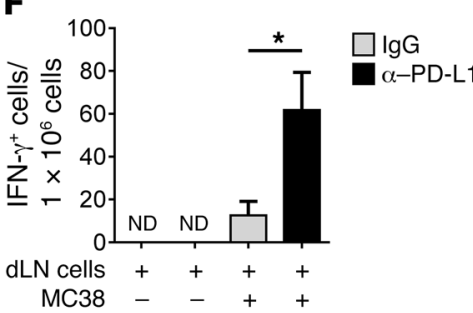

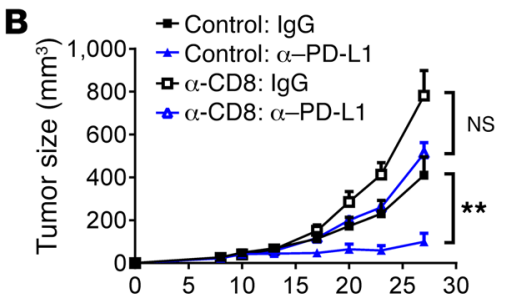

Days after tumor inoculation

D

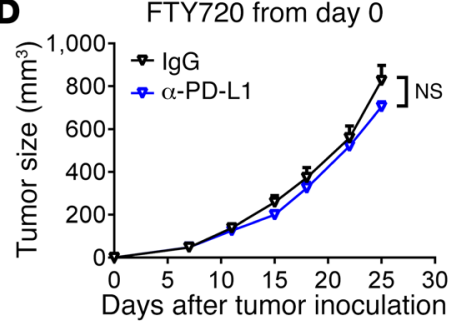

$\mathrm{c}$

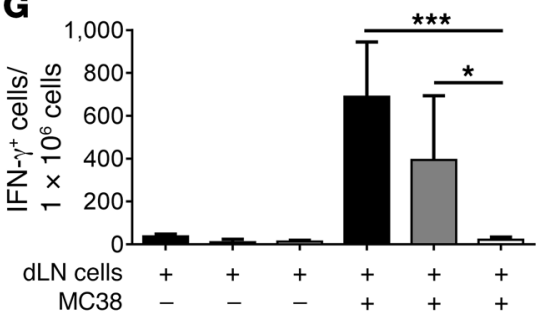

E

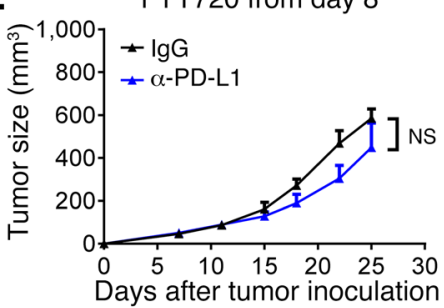

No FTY720

FTY720 from day 8

$\square$ FTY720 from day 0

Figure 3. Preexisting TIL is insufficient for the effects of PD-L1 blockade. (A) B6.Rag ${ }^{-/-}$mice $(n=5)$ were inoculated with $1 \times 10^{6} \mathrm{MC} 38$ cells. After tumors were established, mice were treated with $200 \mu \mathrm{g}$ anti-PD-L1 on days 8 and 11. Tumor growth was measured twice a week. (B) C57BL/6 mice $(n=5)$ were inoculated with $1 \times 10^{6} \mathrm{MC} 38$ cells. Mice were treated with $200 \mu \mathrm{g}$ anti-PD-L1 on days 8 and 11 . For CD8 ${ }^{+} \mathrm{T}$ cell depletion, mice were treated with $200 \mu \mathrm{g}$ anti-CD8 on days 8,11 , and 14. (C-E) MC38 tumor-bearing mice ( $n=5$ per group) were treated with $200 \mu \mathrm{g}$ lgG or anti-PD-L1 on days 8 and 11 . Mice were also treated with control (C) or FTY720 from day 0 (D) or day 8 (E). (F) MC38 tumor-bearing mice were treated with IgC or anti-PD-L1 ( $n=3$ per group). Two days later, dLN were isolated and single-cell suspensions were prepared. Cells were cocultured with or without MC38 cells for 2 days. IFN- $\gamma^{+}$cells were measured by ELISPOT. (C) MC38 tumor-bearing mice were treated with anti-PD-L1 and FTY720 as in C-E. Three days after anti-PD-L1 treatment, dLNs were isolated. ELISPOT assay was performed. Data indicate mean \pm SEM and are representative of 2 independent experiments. Statistical analysis was performed using an unpaired Student's 2 -tailed $t$ test. ${ }^{*} P<0.05$; ${ }^{* *} P<0.01 ;{ }^{* *} P<0.001$. ND, not detectable.

er. Molecular in vivo imaging with radiolabeled anti-PD-L1 Ab allows noninvasive real-time detection of PD-L1 expression (20). To address whether PD-L1 on tumor or immune cells is essential for $\mathrm{Ab}$ targeting, anti-PD-L1 Ab was labeled with ${ }^{8} \mathrm{Zr}$ for tracking. ${ }^{89} \mathrm{Zr}$-anti-PD-L1 $\mathrm{Ab}$ bound to PD-L1 with an affinity similar to that of unconjugated $\mathrm{Ab}$ (data not shown). To image PD-L1 in vivo, MC38 tumor-bearing mice were injected with ${ }^{89} \mathrm{Zr}$-antiPD-L1. Whole-body PET/CT was performed 1, 2, 3, and 6 days post injection (d.p.i) (Figure 2A and Supplemental Figure 3A). At 1 d.p.i., strong signals of $\mathrm{Ab}$ were detected in the liver, spleen, heart, and tumor tissues. Twenty-four hours later, $\mathrm{Ab}$ concentrations in other organs gradually reduced, while concentrations in tumor tissues remained high (Figure 2B and Supplemental Figure $3 \mathrm{~B}$ ). Therefore, anti-PD-L1 Ab targeting to tumor tissues can be imaged in real time. We further tested the impact of tumor cell-expressed PD-L1 on Ab targeting. Interestingly, the accumulations of $\mathrm{Ab}$ in tumor tissues were similar between WT and PD-L1 ${ }^{-/}$MC38 tumors (Figure 2, B and C, and Supplemental Figure 3 C) or A20 tumors (Figure 2D and Supplemental Figure 3, D and E). Together, these data support the notion that tumor cellexpressed PD-L1 is dispensable for Ab targeting.
Preexisting tumor-infiltrating lymphocyte is insufficient for the antitumor effects of PD-L1 blockade. To test whether $\mathrm{T}$ cells are essential for anti-PD-L1-mediated tumor regression, immunodeficient mice were utilized to test the role of lymphocytes. Data suggest that $\mathrm{T}$ cells are essential in anti-PD-L1-mediated tumor regression, as immunodeficient mice did not respond to PD-L1 blockade therapy (Figure 3A and Supplemental Figure 4A). Consistent results have been obtained when $\mathrm{CD} 8^{+} \mathrm{T}$ cells were depleted by Abs (Figure 3B and Supplemental Figure 4B). If PD-L1 on tumor cells is not essential, the role of PD-L1 on antigen-presenting cells (APCs), inside or outside tumor tissues, might be underestimated in suppressing $\mathrm{T}$ cell priming or reactivation. To test whether PD-1/PD-L1 interaction is essential for suppressing T cell activation, we utilized FTY720 to block activated lymphocyte trafficking to tumor tissues. FTY720 is a small molecule analogue of sphingosine 1-phosphate (S1P) (17). Treating mice with FTY720 induces the internalization and degradation of the S1P receptor, thereby preventing lymphocyte egresses from the secondary lymphoid tissues. FTY720 treatment alone has no effects on tumor growth (Supplemental Figure 4C). When mice were treated with FTY720 on the day of tumor inoculation, 

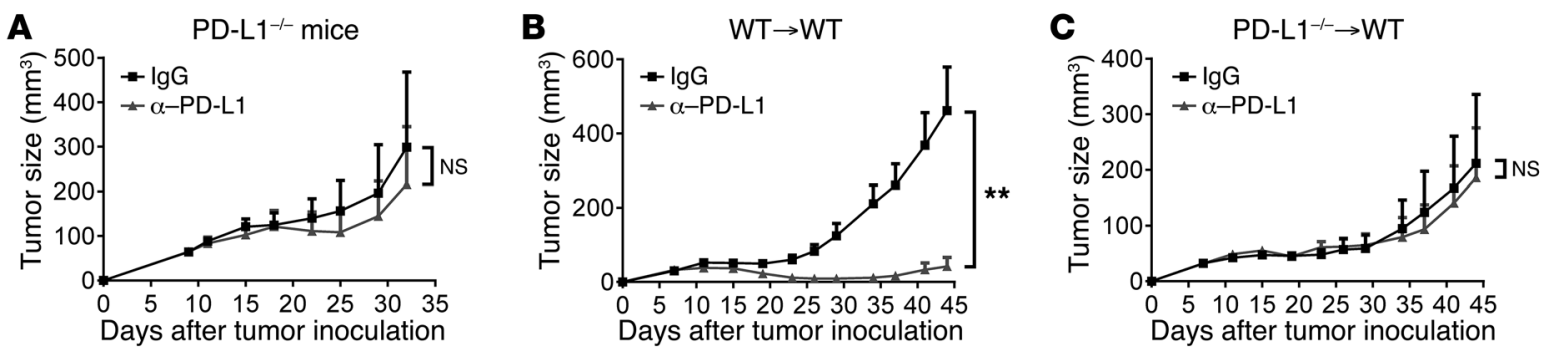

Figure 4. Host PD-L1 is essential for the responses to PD-L1 blockade. (A) PD-L1/- mice were inoculated with $1 \times 10^{6}$ WT MC38 cells. Mice were treated with $200 \mu \mathrm{g}$ IgG or anti-PD-L1 on days 9 and $12(n=4)$. Tumor growth was measured twice weekly. (B and C) C57BL/6 mice were reconstituted with bone marrow cells from WT (B) or PD-L1/- (C) mice. Eight weeks after reconstitution, mice were inoculated with $1 \times 10^{6} \mathrm{MC} 38$ cells and treated with $200 \mu \mathrm{g}$ anti-PD-L1 on days 7, 10, and 13 ( $n=7$ for WT, $n=5$ for PD-L1 ${ }^{-1-}$ ). Data indicate mean \pm SEM and are from a pool of 2 independent experiments. Statistical analysis was performed using an unpaired Student's 2-tailed $t$ test. ${ }^{* *} P<0.01$.

lymphocyte trafficking to tumor tissues was greatly reduced (Supplemental Figure 4, D-F). Such treatment completely abrogated the antitumor effects (Figure 3, C and D). When FTY720 was applied on the same day PD-L1 blockade therapy started, it only blocked further lymphocytes from entering tumors. Preexisting tumor-infiltrating lymphocytes (TILs) were still being activated and stayed inside the tumor. Blocking lymphocyte trafficking after 8 days of inoculation readily reduced the majority of the antitumor effects (Figure 3E). The same results were observed in the A20 model (Supplemental Figure 4, G and H). These data suggest that reversing PD-L1 suppression on preexisting TILs is not sufficient for antitumor effects of PD-L1 blockade. In order to control tumor growth, more $\mathrm{T}$ cells need to be activated outside the tumor, likely within dLNs, and then trafficked into tumor tissues to contribute to overall responses. These data further explain why PD-L1 on tumor cells is not necessarily essential for anti-PD-L1-mediated tumor regression.

Since our data suggested that $\mathrm{T}$ cell activation outside the tumor may also be important, we then sought to evaluate $\mathrm{T}$ cell activation after PD-L1 blockade. Anti-PD-L1 induced LN swelling within 48 hours after treatment, indicating lymphocyte proliferation in dLNs in the early phase of PD-L1 blockade (Supplemental Figure 4, I and J). To test whether the responses were tumor specific, dLNs were isolated from mice. Single-cell suspensions were prepared and cocultured with tumor cells in vitro. The presence of tumor cells dramatically increased $\mathrm{T}$ cell activation in anti-PD-L1-treated, but not IgG-treated, mice, indicating a tumor-specific response (Figure 3F). FTY720 treatment abrogated $\mathrm{T}$ cell activation in $\mathrm{ALN}$ (Figure 3G). In summary, these data suggest that $\mathrm{PD}-\mathrm{L} 1$ could suppress $\mathrm{T}$ cell priming or reactivation in dLN. Blocking PD-L1 signaling in dLN might contribute to $\mathrm{Ab}$-mediated tumor control.

$P D-L 1$ in host APCs is essential for the responses to PD-L1 blockade therapy. PD-L1 can be expressed in many cells, including tumor and host cells (21). Our data suggested that PD-L1 expressed in tumor cells is not essential for the antitumor effects of PD-L1 blockade (Figure 1, F and H). We then sought to determine whether PD-L1 expressed in host cells is essential. To test this hypothesis, PD-L1-deficient mice were inoculated with WT MC38 tumor. After tumors were established, mice were treated with anti-PD-L1 or control IgG. Intriguingly, MC38 tumors that grew in PD-L1-deficient mice did not respond to anti-PD-L1 at all (Figure 4A). Since
PD-L1 can be expressed on both hematopoietic cells and nonhematopoietic cells, we constructed bone marrow chimeric mice to further determine whether PD-L1 expressed on bone marrowderived cells or stromal cells of the host is required. In mice reconstituted with WT bone marrow, MC38 tumors responded well to anti-PD-L1 (Figure 4B). In contrast, tumors in mice reconstituted with PD-L1'-- bone marrow cells did not respond (Figure 4C). The same results were observed in the E.G7 tumor model (Supplemental Figure 5, A-C). These data suggest that PD-L1 expressed in host bone marrow-derived cells plays more important roles.

To further nail down which cell or cells are essential, we depleted macrophages or MDSCs with Abs. However, neither population is essential for the responses (Figure 5A and Supplemental Figure 5, D and E). Depleting phagocytes by clodronate liposome had no effects either (data not shown). Since there were no effects depleting a single subset, we sought to deplete PD-L1+ cells from the entire myeloid compartment by constructing CD11b-diphtheria toxin receptor (CD11b-DTR)/PD-L1 $1 /$ mixed bone marrow chimeric mice. Diphtheria toxin (DT) administration eliminated the majority of CD11 ${ }^{+} \mathrm{PD}-\mathrm{L}^{+}$cells, but did not affect CD11 ${ }^{+} \mathrm{PD}-\mathrm{L} 1^{-}$ cells (Figure $5 \mathrm{~B}$ ). Without DT treatment, tumors responded to anti-PD-L1 (Figure 5C). The response completely disappeared in the presence of DT (Figure 5D). Together, these data suggest that PD-L1 in myeloid cells is essential for responses.

To further dissect the mechanisms of PD-L1 blockade, bone marrow-derived macrophages (BMDMs) from $\mathrm{WT}$ or $\mathrm{PD}-\mathrm{L1}^{-/}$ mice were cocultured with $2 \mathrm{C}$ T cells in the presence of SIY peptide. PD-L1-deficient macrophages induced significantly higher T cell activation, as evaluated by both cytokine production (Figure 5E and Supplemental Figure 5, F and G) and activation marker (Figure 5F). To test the effects of anti-PD-L1 in a system that better recapitulates the tumor microenvironment, DCs and $\mathrm{T}$ cells were isolated from dLNs of tumor-bearing mice. Cells were cocultured for 4 days in the presence of anti-PD-L1 or control Ab. PD-L1 blockade significantly increased the IFN- $\gamma$ production by $\mathrm{T}$ cells (Figure $5 \mathrm{G}$ ). Collectively, these data suggest that PD-L1 expressed in APCs negatively regulates $\mathrm{T}$ cell function. Blocking PD-L1 signaling releases these inhibitions, leading to better $\mathrm{T}$ cell activation.

\section{Discussion}

Checkpoint blockade therapies, including PD-L1 blockade, have shown unprecedented durable responses in some patients with 

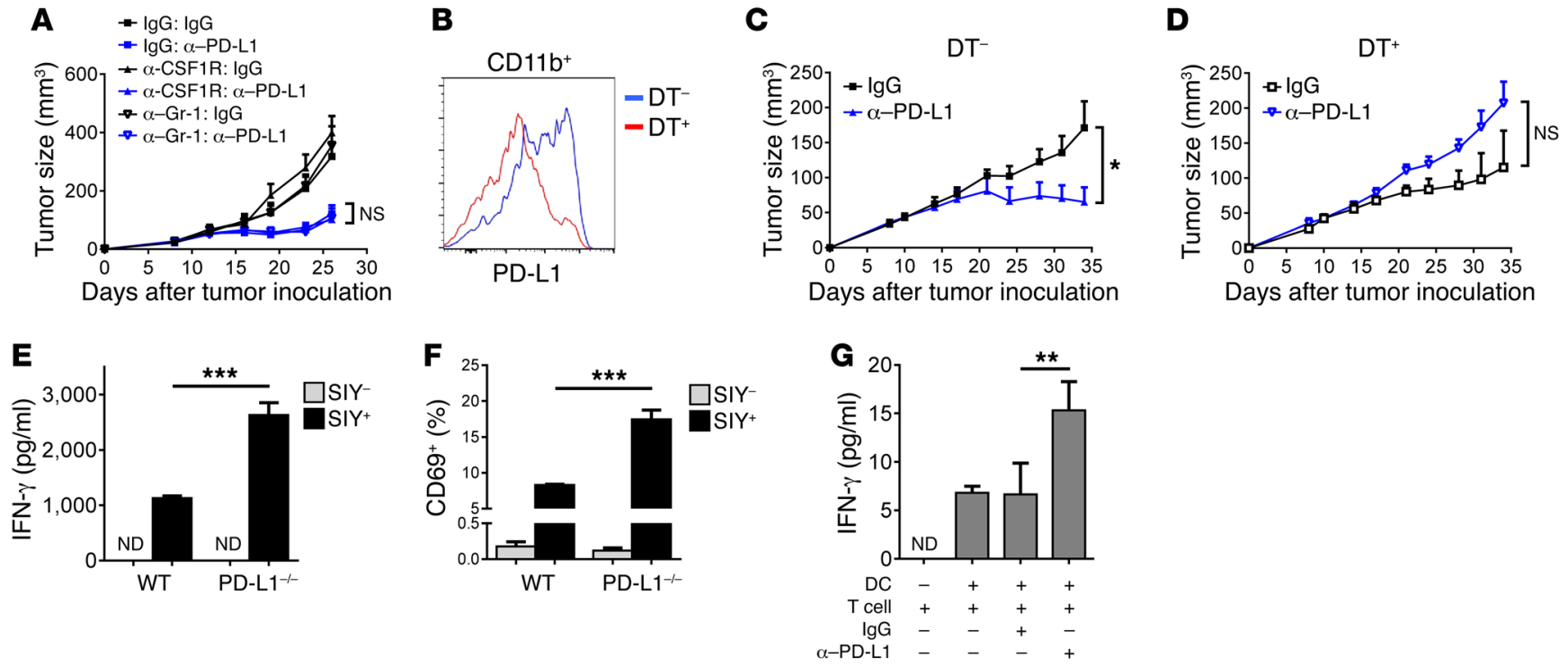

Figure 5. PD-L1 signaling in myeloid cells harnesses antitumor immunity. (A) C57BL/6 mice $(n=5)$ were inoculated with $1 \times 10^{5}$ WT MC38 cells and treated with $200 \mu \mathrm{g}$ IgG or anti-PD-L1 on days 9 and 12. For cell depletion, $300 \mu \mathrm{g}$ IgG, anti-CSF1R, or anti-Gr-1 Abs were injected from day 8. (B-D) C57BL/6 mice were reconstituted with mixed bone marrow cells from CD11b-DTR and PD-L1/- mice. Eight weeks after reconstitution, mice were inoculated with $1 \times 10^{6}$ MC38 cells and treated with $200 \mu \mathrm{g}$ anti-PD-L1 treatment on days 11 and $14(n=5)$. DT was injected intraperitoneally every other day from day 11 . Twentyfour hours after the second DT injection, PD-L1 levels in tumor-infiltrating-CD11 b+ cells were measured by flow cytometry (B). Tumor growth without (C) or with (D) DT was measured twice a week. (E and F) BMDMs from WT or PD-L1/- mice were loaded with SIY peptide, then cocultured with $2 \mathrm{C} \mathrm{T}$ cells for 3 days. (E) IFN- $\gamma$ levels in culture supernatant were measured by CBA. (F) T cell activation was evaluated by flow cytometry. (C) DCs and CD8 $8^{+}$cells were isolated from dLNs of MC38 tumor-bearing mice and cocultured for 4 days. Anti-PD-L1 or control lgG was added into medium at a concentration of $10 \mu \mathrm{g} / \mathrm{ml}$. Cell culture supernatants were harvested, and IFN- $\gamma$ levels were measured by CBA. Data indicate mean \pm SEM and are representative of 2 (A, B, G) or 3 (E, F), or a pool of $2(\mathbf{C}, \mathbf{D})$ independent experiments. Statistical analysis was performed using an unpaired Student's 2-tailed $t$ test. ${ }^{*} P<0.05$; ${ }^{* *} P<0.01$; ${ }^{* * *} P<0.001$.

a variety of cancers. PD-L1 can be expressed in many different cells besides tumor cells. However, the essential or sufficient role of PD-L1 on tumor versus nontumor cells for immune evasion is still unclear $(1,22,23)$. Recently, the importance of PD-L1 on tumor versus host cells during tumor growth has been intensively debated (24-27). In one study, the role of PD-L1 during tumor formation was studied (25). It is suggested that both hostand tumor-PD-L1 contribute to immune suppression during the establishment of tumors (25). In contrast, another study shows that PD-L1 in tumor cells is sufficient for immune evasion (26). In the latter study, a lower cell number was used to slow tumor growth and induce a more robust adaptive immune response. In such a situation, PD-L1 on tumor cells may play a dominant role to prevent tumor clearance in an early phase (26). In addition, it is worth noting that different studies utilized different mouse strains and reagents. Nevertheless, these studies focus on the roles of PD-L1 signaling during tumor establishment. The contributions of PD-L1 signaling during checkpoint blockade therapy, especially the cell populations that respond to PD-L1, is not well defined. In our current study, we found that PD-L1 in tumor cells is dispensable for the responses to PD-L1 blockade therapy after tumor establishment in 3 different models (MC38, A20, and E.G7). Anti-PD-L1 Abs accumulate in tumor tissues regardless of the status of tumor-expressed PD-L1. By using a mixed bone marrow chimera model, we further show that PD-L1 expressed in myeloid cells is essential. PD-L1 is not only highly expressed in myeloid cells, but also contributes to the inhibition of $\mathrm{T}$ cell activation. Blocking PD-L1 signaling by
$\mathrm{Ab}$ releases such inhibition, leading to better $\mathrm{T}$ cell activation. Our data suggest that blocking PD-L1 signaling in myeloid cells is essential for tumor control.

Some recent retrospective clinical studies have shown a correlation between tumor cell-expressed PD-L1 and positive responses to checkpoint blockade therapy $(2,23)$. However, clinical responses were also observed in some patients that were negative for PD-L1 in tumors $(9,10)$. It is unclear why negative patients can still respond. First, it is possible that there might be temporal and spatial heterogeneity of PD-L1 expression in tumor tissues. A false-negative result might result if only a small piece of previously collected specimen is stained (28). Second, another possibility is that several $\mathrm{Ab}$ clones with different cutoff values have been utilized to evaluate PD-L1 expression in different studies (29). Some specimens negative in one test might turn out to be positive in other assays. Third, since PD-L1 expression can be induced during inflammatory responses, it is possible that PD-L1 in some negative tumors can become positive after treatment (19). Here, our study raises the fourth possibility, that high PD-L1 expression outside tumor tissues can also contribute to overall immune suppression. Some patients may have high PD-L1 expression in the myeloid compartment, either inside tumor or lymphoid tissues, which is responsible for their responses. A recent study using a different tumor model and human tissues also showed that PD-L1 is highly expressed in APCs and correlates with type I IFN signaling for combined therapy resistance (30). However, it is worth noting that our study does not rule out the possibility that tumor-derived PD-L1 
could play important roles in some cases, especially during the early phase of tumor establishment or when PD-L1 is constitutively highly expressed on tumor cells. Early studies have shown that ectopic expression of PD-L1 in tumor cells is able to promote tumor evasion in vivo, which can be subverted by PD-L1 blockade therapy $(5,6)$. However, few patients have high tumor cell-expressed PD-L1 in the absence of PD-L1 on host cells (7). In fact, PD-L1 expression is mostly detected around tumors and inflammatory margins $(7,8)$. Therefore, PD-L1 on nontumor cells, either in tumors or lymphoid tissues, may contribute to overall responses in most cases.

During antitumor immune responses, APCs uptake tumor antigens from tumor tissues, migrate to $\mathrm{dLN}$, and present antigens to $\mathrm{T}$ cells, leading to $\mathrm{T}$ cell activation $(31,32)$. After that, activated $\mathrm{T}$ cells traffic to tumor tissues for destruction. PD-L1 signaling plays an important role in inhibiting T cell functions. In the absence of host PD-L1, we observed that tumors grew slower in $\mathrm{PD}-\mathrm{L1}^{-/}$host or chimeric mice reconstituted with $\mathrm{PD}-\mathrm{L1}^{-/}$ bone marrow (Figure 4, A and C). In contrast, we didn't see significant differences in tumor growth between WT and PD-L1deficient tumors after tumors were established. These observations are consistent with our conclusion that PD-L1 signaling on the host is more important. In the presence of PD-L1 blockade, responding $\mathrm{T}$ cells can come from 2 major sources. Some are preexisting $\mathrm{T}$ cells, while others are newly activated $\mathrm{T}$ cells migrating from $\mathrm{dLN}$ to the tumor. It has been shown that some immunotherapies, such as CTLA- 4 and PD-L1 dual blockade, are able to expand $\mathrm{T}$ cells inside the tumor. In those cases, blocking $\mathrm{T}$ cell trafficking has limited effects (33). In contrast, our data show that blocking $\mathrm{T}$ cell trafficking reduces the majority of the antitumor effects of PD-L1 mono-blockade. These data suggest that PD-L1 blockade therapy may require $\mathrm{T}$ cell activation/ reactivation both inside and outside the tumor (Figure 3, C-E). Consistent with our observations, a recent study shows that surgical dLN ablation completely abrogates the antitumor effects of PD-L1 blockade (34). Together, our data suggest that the impact of PD-L1 blockade outside the tumor needs to be revisited. These findings further demonstrate that PD-L1 expressed in APCs, even outside tumor tissues, plays an essential role in checkpoint blockade therapy, providing new insight into the mechanisms and potential biomarkers of checkpoint blockade therapy.

\section{Methods}

Mice. C57BL/6, BALB/c, Rag1 ${ }^{-/}$, CD11b-DTR, and NSG mice were purchased from the Mouse Breeding Core at UT Southwestern Medical Center or the Jackson Laboratory. PD-L1 ${ }^{-/-}$mice were provided by L. Chen (Yale University, New Haven, Connecticut, USA). All mice were maintained under specific pathogen-free conditions at UT Southwestern Medical Center.

Cell lines and reagents. MC38, A20, and E.G7 cells were purchased from ATCC. Cells were cultured in $5 \% \mathrm{CO}_{2}$ and maintained in vitro in DMEM or RPMI 1640 medium supplemented with 10\% FBS (Sigma-Aldrich), nonessential amino acids, $100 \mathrm{U} / \mathrm{ml}$ penicillin, and 100 $\mu \mathrm{g} / \mathrm{ml}$ streptomycin. Anti-PD-L1 (10F.9G2), anti-PD-1 (RMP1-14), anti-CSF1R (AFS98), and anti-Gr-1 (RB6-8C5) Abs were purchased from BioXCell. Anti-CD8 (YTS) was produced in house. FTY720 and DT were purchased from Sigma-Aldrich.
Generation of PD-L1-deficient cell lines. PD-L1 in MC38 or A20 cells was knocked out by CRISPR/Cas9 technology. The guide sequences (5'-GACTTGTACGTGGTGGAGTA-3', designed by CRISPR DESIGN; http://crispr.mit.edu) and EGFP gene were cloned into lentiCRISPR v2 plasmid (Addgene, catalog 52961). Thirty hours after transfection, EGFP-positive cells were sorted and subcloned by flow cytometry. Two weeks later, cells were treated with or without

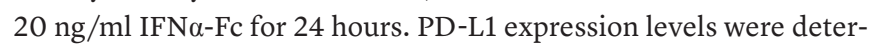
mined by FACS. Cell clones without PD-L1 or EGFP expression were used for the following studies.

Flow cytometric analysis. Flow cytometry was performed as described (17). Briefly, cells were blocked with anti-CD16/32 (anti-Fc $\gamma I I I /$ II receptor, clone 2.4G2) for 20 minutes. Cells were then incubated with Abs for 30 minutes at room temperature. Details of fluorescent-labeled Abs are listed (Supplemental Table 1). Samples were analyzed on an LSR-II (BD Biosciences) or CytoFLEX (Beckman Coulter) flow cytometer. Data were analyzed by FlowJo software (TreeStar).

Mouse PET/CT imaging. PET/CT imaging was performed using the Siemens Inveon PET/CT Multi-Modality system (Siemens Medical Solutions), as described previously (35). Briefly, tumor-bearing mice were injected intravenously with $50 \mu \mathrm{Ci}$ of ${ }^{89} \mathrm{Zr}$-radiolabeled deferoxamine-conjugated anti-PD-L1 (10F.9G2) Abs. On 1, 2, 3, and 6 d.p.i., static PET images were acquired. PET images were reconstructed using the 3D Ordered Subsets Expectation Maximization (OSEM3D/ MAP) algorithm. CT images were acquired immediately after PET. PET and CT images were coregistered in the Inveon Acquisition Workplace for analysis. The target activity was calculated as percentage of injected dose per gram (\%ID/g).

Tumor growth and treatments. MC38 cells $\left(1 \times 10^{6}\right)$ were subcutaneously injected into the right flank of mice. Mice were randomized to treatment groups when tumors reached certain sizes and treated intraperitoneally with $200 \mu \mathrm{g}$ anti-PD-L1 (10F.9G2) on days 8 and 11. Tumor volumes were measured twice weekly and calculated as length $\times$ width $\times$ height $/ 2$. Mice were sacrificed when tumor size was greater than $500 \mathrm{~mm}^{3}$. To inhibit lymphocyte trafficking, mice were injected intravenously with $25 \mu \mathrm{g}$ FTY720 on day 0 or 8 after tumor inoculation. Five micrograms of FTY720 were given every day to maintain inhibition. For the A20 model, $3 \times 10^{6}$ cells were inoculated and mice were treated on days 9, 12, and 15. For macrophage and MDSC depletion, mice were treated intraperitoneally with $300 \mu \mathrm{g}$ Ab every other day (anti-CSF1R) or every 4 days (anti-Gr-1).

Generation of bone marrow chimeras. WT C57BL/6 mice were lethally irradiated at $10 \mathrm{~Gy}$. Seven to eight hours later, $2 \times 10^{6}$ bone marrow cells from WT or $\mathrm{PD}-\mathrm{L1}^{-/-}$mice were transferred into irradiated mice. Mice were used for experiments 8 weeks after reconstitution. To generate mixed bone marrow chimera, bone marrow cells from CD11b-DTR and $\mathrm{PD}-\mathrm{L1}^{-/-}$mice were mixed 1:1 before adoptive transfer.

Cell isolation from tissues. Tissues were collected and analyzed as previously described (17). Briefly, tumor tissues were collected, cut into small pieces, and resuspended in RPMI 1640 medium with $1.5 \mathrm{mg} / \mathrm{ml}$ type I collagenase and $100 \mu \mathrm{g} / \mathrm{ml}$ DNase I. Tumors were digested for 45 minutes in a $37^{\circ} \mathrm{C}$ shaking incubator, then passed through a $70-\mu \mathrm{m}$ cell strainer to make single-cell suspensions. Similarly, spleens and dLNs were digested for 30 or 15 minutes, respectively, before passing through a cell strainer.

ELISPOT. Draining LNs from MC38 tumor-bearing mice were isolated and single-cell suspensions were prepared. LN cells and 
irradiated tumor cells were cocultured at a ratio of 10:1 for 36 hours. ELISPOT assay was performed using an IFN- $\gamma$ ELISPOT assay kit (BD Biosciences) according to the manufacturer's instruction. Spots were enumerated by ImmunoSpot Analyzer (CTL).

In vitro and ex vivo culture and function assays. Bone marrow cells were obtained from WT or PD-L1 $1^{-/}$mice. Cells were cultured in 10 -cm petri dishes in an RPMI 1640 medium containing $10 \% \mathrm{FBS}$ and $55 \mu \mathrm{mol} / 1$ 2-mercaptoethanol, supplemented with $20 \mathrm{ng} / \mathrm{ml}$ mouse M-CSF. Fresh medium with M-CSF was added every 3 days. BMDMs were harvested and cocultured with $2 \mathrm{C}$ T cells at a ratio of $1: 10$. SIY peptide was added at a concentration of $5 \mu \mathrm{g} / \mathrm{ml}$. Cells were harvested after 72 hours and analyzed by flow cytometry. Cytokine levels in culture supernatants were measured using a cytometric bead array (CBA) kit (BD Biosciences).

DCs were purified from dLNs of MC38 tumor-bearing mice by EasySep Mouse CD11c Positive Selection Kit (STEMCELL Technologies). $\mathrm{CD}^{+} \mathrm{T}$ cells were purified from the same mice using the EasySep Mouse $\mathrm{CD}^{+} \mathrm{T}$ cell Isolation Kit (negative selection, STEMCELL Technologies). DC and T cells were cocultured at a ratio of 1:10 for 7 days. Cytokine levels were measured by CBA.

Statistics. For in vivo studies, sample size was estimated using data from others' and our previous studies to see an effect at the $P<0.05$ significance levels. The number of animals and replicates are indicated in each figure legend. All data are presented as mean \pm SEM. Mean values were compared using an unpaired Student's 2-tailed $t$ test. Statistical analysis was performed using GraphPad Prism 6.0 software (GraphPad). Investigators were blinded to the group allocation during the experiment and drug treatment whenever possible.

Study approval. Animal experiment protocols were consistent with NIH guidelines. All studies were approved by the Animal Care and Use Committee of UT Southwestern Medical Center.

\section{Author contributions}

HT and YXF designed experiments, analyzed data, and wrote the manuscript. HT, YL, XL, XQ, RAA, JMT, Y Xin, Y Xiong, KN, and AM performed experiments. WS, GH, and XS analyzed data. MC, $\mathrm{RH}, \mathrm{SMH}$, and JG provided reagents. XS, JQ, and HD contributed to manuscript preparation. HP, HD, and YXF supervised the project.

\section{Acknowledgments}

We thank Changzheng Lu for helpful scientific discussions and Daryl Harmon for help in manuscript preparation. We also thank the UT Southwestern Flow Cytometry Facility, Animal Resources Center, and Whole Brain Microscopy Facility. YXF holds the Mary Nell and Ralph B. Rogers Professorship in Immunology. This work was in part supported by the NIH through National Cancer Institute grants CA141975, Texas CPRIT grant RR150072, and grants from the Chinese Academy of Sciences (XDA09030303) and the Chinese Ministry of Science and Technology (2012ZX10002006 and 2012AA020701) to YXF, NIH/NIAID grant AI 095239 to HD, and a Cancer Research Institute Irvington Fellowship to HT.

Address correspondence to: Haidong Dong, Departments of Urology and Immunology, College of Medicine, Mayo Clinic, 200 First Street SW, Rochester, Minnesota 55905, USA. Phone: 507.284.5482; Email: Dong.Haidong@mayo.edu. Or to: Hua Peng, Chinese Academy of Science Key Laboratory for Infection and Immunity, Institute of Biophysics, Chinese Academy of Sciences, Beijing 100101, China. Phone: 8610.6488.1152; Email: hpeng@moon.ibo.cas.cn. Or to: Yang-Xin Fu, Department of Pathology, UT Southwestern Medical Center, 6000 Harry Hines Blvd., Dallas, Texas 75235, USA. Phone: 214.648.6538; Email: Yang-Xin.Fu@UTSouthwestern.edu.
1. Zou W, Wolchok JD, Chen L. PD-L1 (B7-H1) and PD-1 pathway blockade for cancer therapy: Mechanisms, response biomarkers, and combinations. Sci Transl Med. 2016;8(328):328rv4.

2. Topalian SL, et al. Safety, activity, and immune correlates of anti-PD-1 antibody in cancer. $N$ Engl JMed. 2012;366(26):2443-2454.

3. Brahmer JR, et al. Safety and activity of antiPD-L1 antibody in patients with advanced cancer. N Engl J Med. 2012;366(26):2455-2465.

4. Sznol M, Chen L. Antagonist antibodies to PD-1 and B7-H1 (PD-L1) in the treatment of advanced human cancer. Clin Cancer Res. 2013;19(5):1021-1034.

5. Iwai Y, Ishida M, Tanaka Y, Okazaki T, Honjo T, Minato N. Involvement of PD-L1 on tumor cells in the escape from host immune system and tumor immunotherapy by PD-L1 blockade. Proc Natl Acad Sci U S A. 2002;99(19):12293-12297.

6. Hirano F, et al. Blockade of B7-H1 and PD-1 by monoclonal antibodies potentiates cancer therapeutic immunity. Cancer Res. 2005;65(3):1089-1096.

7. Taube JM, et al. Colocalization of inflammatory response with B7-h1 expression in human melanocytic lesions supports an adaptive resistance mechanism of immune escape. Sci Transl Med. 2012;4(127):127ra37.

8. Tumeh PC, et al. PD-1 blockade induces respons- es by inhibiting adaptive immune resistance. Nature. 2014;515(7528):568-571.

9. Powles T, et al. MPDL3280A (anti-PD-L1) treatment leads to clinical activity in metastatic bladder cancer. Nature. 2014;515(7528):558-562.

10. Robert $C$, et al. Nivolumab in previously untreated melanoma without BRAF mutation. $N$ Engl Med. 2015;372(4):320-330.

11. Chen DS, Irving BA, Hodi FS. Molecular pathways: next-generation immunotherapy--inhibiting programmed death-ligand 1 and programmed death-1. Clin Cancer Res. 2012;18(24):6580-6587.

12. Postow MA, Callahan MK, Wolchok JD. Immune Checkpoint Blockade in Cancer Therapy. JClin Oncol. 2015;33(17):1974-1982.

13. Baumeister SH, Freeman GJ, Dranoff G, Sharpe AH. Coinhibitory Pathways in Immunotherapy for Cancer. Annu Rev Immunol. 2016;34:539-573.

14. Blank C, et al. PD-L1/B7H-1 inhibits the effector phase of tumor rejection by $\mathrm{T}$ cell receptor (TCR) transgenic CD8+ T cells. Cancer Res. 2004;64(3):1140-1145.

15. Nguyen LT, Ohashi PS. Clinical blockade of PD1 and LAG3--potential mechanisms of action. Nat Rev Immunol. 2015;15(1):45-56.

16. Nguyen DP, Thalmann GN. Contemporary update on neoadjuvant therapy for bladder cancer. Nat Rev Urol. 2017;14(6):348-358.

17. Tang H, et al. Facilitating $\mathrm{T}$ cell infiltration in tumor microenvironment overcomes resistance to PD-L1 blockade. Cancer Cell. 2016;29(3):285-296.

18. Selby MJ, et al. Preclinical development of ipilimumab and nivolumab combination immunotherapy: mouse tumor models, in vitro functional studies, and cynomolgus macaque toxicology. PLoS One. 2016;11(9):e0161779.

19. Chen L, Han X. Anti-PD-1/PD-L1 therapy of human cancer: past, present, and future. J Clin Invest. 2015;125(9):3384-3391.

20. Heskamp S, et al. Noninvasive imaging of tumor PD-L1 expression using radiolabeled anti-PD-L1 antibodies. Cancer Res. 2015;75(14):2928-2936.

21. Shin DS, Ribas A. The evolution of checkpoint blockade as a cancer therapy: what's here, what's next? Curr Opin Immunol. 2015;33:23-35.

22. Curiel TJ, et al. Blockade of B7-H1 improves myeloid dendritic cell-mediated antitumor immunity. Nat Med.2003;9(5):562-567.

23. Herbst RS, et al. Predictive correlates of response to the anti-PD-L1 antibody MPDL3280A in cancer patients. Nature. 2014;515(7528):563-567.

24. Kleinovink JW, Marijt KA, Schoonderwoerd MJA, van Hall T, Ossendorp F, Fransen MF. PD-L1 expression on malignant cells is no prerequisite for checkpoint therapy. Oncoimmunology. 2017;6(4):e1294299.

25. Lau J, et al. Tumour and host cell PD-L1 is required to mediate suppression of anti-tumour 
immunity in mice. Nat Commun. 2017;8:14572.

26. Juneja VR, et al. PD-L1 on tumor cells is sufficient for immune evasion in immunogenic tumors and inhibits CD8 T cell cytotoxicity. JExp Med. 2017;214(4):895-904.

27. Noguchi T, et al. Temporally distinct PD-L1 expression by tumor and host cells contributes to immune escape. Cancer Immunol Res. 2017;5(2):106-117.

28. Taube JM, et al. Association of PD-1, PD-1 ligands, and other features of the tumor immune microenvironment with response to anti-PD-1 therapy. Clin Cancer Res. 2014;20(19):5064-5074.

29. Garber K. Predictive biomarkers for check- points, first tests approved. Nat Biotechnol. 2015;33(12):1217-1218.

30. Benci JL, et al. Tumor interferon signaling regulates a multigenic resistance program to immune checkpoint blockade. Cell.2016;167(6):1540-1554.e12.

31. Tang H, Qiao J, Fu YX. Immunotherapy and tumor microenvironment. Cancer Lett. 2016;370(1):85-90.

32. Salmon $\mathrm{H}$, et al. Expansion and activation of CD103 (+) Dendritic cell progenitors at the tumor site enhances tumor responses to therapeutic PD-L1 and BRAF inhibition. Immunity. 2016;44(4):924-938.

33. Spranger S, Koblish HK, Horton B, Scherle PA,
Newton R, Gajewski TF. Mechanism of tumor rejection with doublets of CTLA-4, PD-1/PD-L1, or IDO blockade involves restored IL-2 production and proliferation of CD8(+) T cells directly within the tumor microenvironment. J Immunother Cancer. 2014;2:3.

34. Chamoto K, et al. Mitochondrial activation chemicals synergize with surface receptor PD-1 blockade for T cell-dependent antitumor activity. Proc Natl Acad Sci U S A. 2017;114(5):E761-E770.

35. Hao G, et al. Serial non-invasive assessment of antibody induced nephritis in mice using positron emission tomography. PLoS One. 2013;8(2):e57418. 